\title{
Transitions in the Ergodicity of Subrecoil-Laser-Cooled Gases
}

\author{
Eli Barkai®i \\ Department of Physics, Institute of Nanotechnology and Advanced Materials, Bar-Ilan University, \\ Ramat-Gan 52900, Israel \\ Günter Radons (1) \\ Institute of Physics, Chemnitz University of Technology, 09107 Chemnitz, Germany \\ and Institute of Mechatronics, 09126 Chemnitz, Germany \\ Takuma Akimoto (1) \\ Department of Physics, Tokyo University of Science, Noda, Chiba 278-8510, Japan
}

(Received 11 April 2021; accepted 26 August 2021; published 30 September 2021)

\begin{abstract}
With subrecoil-laser-cooled atoms, one may reach nanokelvin temperatures while the ergodic properties of these systems do not follow usual statistical laws. Instead, due to an ingenious trapping mechanism in momentum space, power-law-distributed sojourn times are found for the cooled particles. Here, we show how this gives rise to a statistical-mechanical framework based on infinite ergodic theory, which replaces ordinary ergodic statistical physics of a thermal gas of atoms. In particular, the energy of the system exhibits a sharp discontinuous transition in its ergodic properties. Physically, this is controlled by the fluorescence rate, but, more profoundly, it is a manifestation of a transition for any observable, from being an integrable to becoming a nonintegrable observable, with respect to the infinite (non-normalized) invariant density.
\end{abstract}

DOI: 10.1103/PhysRevLett.127.140605

Laser-cooled atoms are important for fundamental and practical applications [1-3]. It is well known that Lévy statistics describes the unusual properties of the cooling processes [4-11]. For subrecoil-laser cooling, a special atomic trap in momentum space is engineered. The most efficient cooling is found when a mean trapping time, defined more precisely below, diverges [6]. The fact that the characteristic time diverges implies that the ergodic properties of these systems must differ from those of standard gases $[6,12,13]$. Ergodicity is a fundamental aspect of statistical mechanics that implies that the time and ensemble averages coincide. This is found when the measurement time is made long compared to the timescale of the dynamics. However, in the context of subrecoil-laser cooling, this time diverges, and, hence, no matter how long one measures, deviations from standard ergodic theory are prominent. Given that lasers replace heat baths in many cooling experiments, what are the ergodic properties of the system? In other words, what replaces the usual ergodic statistical framework? Our goal is to show how tools of infinite ergodic theory describe the statistical properties of the ensemble and corresponding time averages of the subrecoil-laser-cooled atoms.

Infinite ergodic theory was investigated by mathematicians [14-16] and more recently in physics [17-32]. In general, infinite ergodic theory deals with a peculiar nonnormalized density, describing the long-time limit of a system, called below the infinite invariant density. Previous works in the field of subrecoil-laser cooling [6,33] foresaw this quasisteady state. We will see how to use this tool to investigate the ensemble averages of physical observables. However, this does not give direct information on the time averages, and here we will develop physical and mathematical insights on the latter. The basic question is how to relate ensemble and time averages, even when ergodicity in its standard formulation is broken. In particular, we investigate the energy of the system. Since the atoms are noninteracting, in a classical thermal setting, the energy of the atoms per degree of freedom would be $k_{B} T / 2$, as a consequence of Maxwell's velocity distribution. At variance with this, we will show that the energy of a subrecoillaser-cooled gas is obtained under certain conditions with a non-normalizable invariant density. A sharp transition is exposed, in the statistical properties of the energy, when the fluorescence rate, given by $R(v) \propto|v|^{\alpha}$ in the vicinity of zero velocity, is varied, more precisely when $\alpha=3$. In Raman subrecoil cooling, $\alpha$ is controlled by the pulse shape, allowing $\alpha$, in principle, to attain any value [6,3436]. Hence, the rich phase diagram of ergodic properties seems to be within reach of experimental investigations. This new type of transition is related to the fact that the energy observable can switch from being an integrable observable, with respect to the infinite density, to being nonintegrable. 
Let $\rho(v, t)$ be the probability density function (PDF) of the speed $v>0$ of the atoms at time $t$. A master equation governs its evolution with typical gain and loss terms:

$$
\frac{\partial \rho(v, t)}{\partial t}=\int_{0}^{\infty}\left[W\left(v^{\prime} \rightarrow v\right) \rho\left(v^{\prime}, t\right)-W\left(v \rightarrow v^{\prime}\right) \rho(v, t)\right] d v^{\prime} .
$$

The transition rate from $v$ to $v^{\prime}$ is $W\left(v \rightarrow v^{\prime}\right)=R(v) f\left(v^{\prime}\right)$. Here, $R(v)=1 / \tau(v)$ is the fluorescence rate, and $f(v)$ denotes the PDF of $v$ after the atom experiences a spontaneous jolt. It is natural to consider the long-time limit of the $\operatorname{PDF} \rho(v, t)$. Considering the steady-state condition $\partial \rho(v, t) / \partial t=0$ gives the time-independent solution $\rho^{*}(v)=\tau(v) f(v) / Z$, where $Z$, if it exists, is a timeindependent constant obtained from the normalization condition. We treat the highly relevant non-normalizable case.

Indeed, for subrecoil-laser cooling, the fluorescence time $\tau(v)$ is given by $\tau(v) \sim c v^{-\alpha}$ for $v \rightarrow 0$, and then $\rho^{*}(v)$ becomes non-normalizable, for $\alpha>1$. As we will see, this non-normalizable function should not be ignored. An analysis of the master equation [37] and following the footsteps of Refs. [6,33] yields

$$
\lim _{t \rightarrow \infty} Z(t) \rho(v, t)=\tau(v) f(v) \equiv \mathcal{I}(v) \quad \text { for } \alpha>1 .
$$

A calculation gives $Z(t)=\pi \Gamma(1+\gamma) f(0) c^{\gamma} t^{1-\gamma} / \sin \pi \gamma$ with $\gamma=1 / \alpha$. In contrast, if $\alpha<1$, the usual normalization $Z=\int_{0}^{\infty} f(v) \tau(v) d v$, is found. For the case under study, $\gamma<1$ or $\alpha>1$, the integration over $\mathcal{I}(v)$ diverges, due to the small $v$ behavior of $\tau(v)$, and, hence, $\mathcal{I}(v)$ is called an infinite invariant density. Note that, on the left-hand side of Eq. (2), we multiply the normalized density $\rho(v, t)$ with a function $Z(t)$ increasing with time; therefore, on the righthand side, we find in the long-time limit a nonnormalizable function. The question is, what is the physical meaning of this state? And how can we use the infinite invariant density to construct a nontrivial ergodic theory for the gas?

One can argue that the infinite density cannot provide the full picture, as it is non-normalizable. Indeed, for long but finite times, the density of the velocity of the particles can be described by two regimes. As the density evolves, it shrinks in its width, peaking at zero velocities. In the inner region, the density $\rho(v, t)$ exhibits a scaling solution, given by [6] $\rho(v, t) \sim t^{\gamma} g\left(v t^{\gamma}\right)$ with

$$
g(x)=\frac{\mathcal{N}}{\gamma x} \exp \left(-\frac{x^{1 / \gamma}}{c}\right) \int_{0}^{x} \exp \left(\frac{z^{1 / \gamma}}{c}\right) d z .
$$

This describes velocities of the order of $1 / t^{\gamma}$, i.e., an inner region of the packet, vanishing for $t \rightarrow \infty$ [37].
The scaling solution [Eq. (3)] and the infinite density [Eq. (2)] are not separable and together yield a complete description. Mathematically, the two solutions match at intermediate velocities [37]. Equation (3) predicts that the full width at half maximum (FWHM) of the velocity PDF decays like $t^{-1 / 2}$ for $\alpha=2$ and as $t^{-1 / 4}$ for $\alpha=4$. These theoretical predictions were indeed observed in the laboratory with cesium [34,36]; see also quantum Monte Carlo simulation and experiments with helium in Refs. $[6,13]$. However, the scaling solution [Eq. (3)] exhibits what might appear as an unphysical feature. Considering the realistic case $\alpha=2$, the scaling function alone predicts that the second moment of the velocity, namely, the kinetic energy, is infinite, due to the fat tail of the scaling function for large $v$, and in this sense the system cannot be considered cold. This issue is cured, using the non-normalized solution [Eq. (2)]. In general, we need to classify observables based on whether they are integrable or nonintegrable with respect to $\mathcal{I}(v)$. These two classes have vastly different ergodic properties, as shown below.

Ensemble and time averages.-Let $v(t)$ be the random velocity process of an atom. We consider generic observables $\mathcal{O}[v(t)]$, and we study their time and ensemble averages. As examples, consider $\mathcal{O}[v(t)]=v^{2}(t)=$ $E_{k}(t)$, which is the kinetic energy when we set $m / 2=1$, and the indicator function $\mathcal{O}[v(t)]=I\left[v_{a}<\right.$ $\left.v(t)<v_{b}\right]=1$ if $v_{a}<v(t)<v_{b}$ and zero otherwise. By definition, the ensemble average is $\langle\mathcal{O}(t)\rangle=$ $\int_{0}^{\infty} \mathcal{O}(v) \rho(v, t) d v$, so using Eq. (2) we find

$$
\lim _{t \rightarrow \infty} Z(t)\langle\mathcal{O}(t)\rangle=\int_{0}^{\infty} \mathcal{I}(v) \mathcal{O}(v) d v<\infty,
$$

showing that the non-normalized density $\mathcal{I}(v)$ is used in the computation of ensemble averages, in a way reminiscent of the standard averaging for equilibrated systems. The only condition is that the observable is integrable with respect to $\mathcal{I}(v)$, namely, that the integral exists. Since $\mathcal{I}(v) \sim v^{-\alpha}$ for small velocity, the kinetic energy is an integrable observable for $\alpha<3$ but nonintegrable otherwise. The critical value $\alpha=3$ or $\gamma=1 / 3$ will mark an ergodicity transition for this observable. In contrast, the indicator function is always an integrable observable, provided that $v_{a}>0$.

A goal of ergodic theories is to relate the ensemble and time averages, denoted with an overline: $\overline{\mathcal{O}}(t)=$ $\int_{0}^{t} \mathcal{O}\left[v\left(t^{\prime}\right)\right] d t^{\prime} / t$ [40]. According to the standard ergodic hypothesis, $\overline{\mathcal{O}}(t) /\langle\mathcal{O}\rangle \rightarrow 1$ in the limit of long measurement times. In our case, the time averages remain random, and we will soon investigate their fluctuations. To start, we consider an ensemble of paths and average over time and then over the ensemble:

$$
\langle\overline{\mathcal{O}}(t)\rangle=\left\langle\frac{1}{t} \int_{0}^{t} \mathcal{O}\left[v\left(t^{\prime}\right)\right] d t^{\prime}\right\rangle=\frac{1}{t} \int_{0}^{t} \int_{0}^{\infty} \mathcal{O}(v) \rho\left(v, t^{\prime}\right) d v d t^{\prime} .
$$


Because we consider the limit of long measurement times, our asymptotic form of the integrand Eq. (2) yields

$\langle\overline{\mathcal{O}}(t)\rangle \sim \frac{1}{t} \int_{0}^{t} d t^{\prime} \frac{\int_{0}^{\infty} \mathcal{O}(v) \mathcal{I}(v) d v}{Z\left(t^{\prime}\right)}=\frac{\int_{0}^{\infty} \mathcal{O}(v) \mathcal{I}(v) d v}{\gamma Z(t)}$,

where we used $0<\gamma<1$. This resembles the standard calculation of time averages when the invariant density is normalizable. We conclude that, for integrable observables and if $\gamma<1$,

$$
\lim _{t \rightarrow \infty} \frac{\langle\overline{\mathcal{O}}(t)\rangle}{\langle\mathcal{O}(t)\rangle}=\frac{1}{\gamma}
$$

Thus, we established a relation between the time average and the ensemble average. The latter is obtained using the infinite density $\mathcal{I}(v)$, and, thus, this invariant density is not merely a tool for the calculation of the ensemble average, but rather it gives also information on the time average. From Eq. (7), when $\gamma \rightarrow 1$ we approach standard ergodic behavior. Physically, this is related to the observation made below, that the mean time between velocity updates diverges for $\gamma<1$.

The time averages are functionals of the stochastic path $v(t)$, and we now develop a machinery to explore their statistical properties. We focus on the kinetic energy observable, due to its physical importance. To analyze it, we recall the stochastic process under study. Initially, we draw $v$ from $f(v)$, and the particle then remains in this state for a random time denoted $\tilde{\tau}$. This time $\tilde{\tau}$ is exponentially distributed with the lifetime $\tau(v)$ that depends on the velocity. The process is then renewed; namely, after waiting for a time $\tilde{\tau}$, we draw a new velocity from $f(v)$ and, hence, a new lifetime, etc. In simulations below, following Ref. [6], we use a uniform PDF $f(v)=1 / v_{\max }$ for $v<$ $v_{\max }$ and $\tau(v)=c v^{-\alpha}$. Using Eqs. (4) and (7),

$\left\langle\bar{E}_{k}(t)\right\rangle \sim \frac{\int_{0}^{v_{\max }} v^{2} \mathcal{I}(v) d v}{\gamma t^{1-\gamma}}=\frac{\sin (\pi \gamma)}{\Gamma(1+\gamma) \pi} \frac{\left(v_{\max }\right)^{3-1 / \gamma}}{3 \gamma-1}\left(\frac{c}{t}\right)^{1-\gamma}$,

where $\bar{E}_{k}(t)=\int_{0}^{t} E_{k}\left(t^{\prime}\right) d t^{\prime} / t$. This holds when the energy is an integrable observable, so $1 / 3<\gamma<1$. In contrast, when $1<\gamma$, we are in the regime of standard ergodic theory. Interestingly, when we take $\gamma \rightarrow 1 / 3$, the prefactor in Eq. (8) diverges and becomes $v_{\max }$ independent. This marks the entry into the phase where the energy is nonintegrable. Having calculated the expectation of the timeaveraged energy with the infinite density $\mathcal{I}(v)$, the real challenge is to obtain the distribution of $\bar{E}_{k}$.

We rewrite the time average $\bar{E}_{k}(t)=\mathcal{S}(t) / t$, where $\mathcal{S}(t)$ is the action. The key idea is to investigate the distribution of the action and with this to infer the ergodic properties of the process. We perform this task with a new form of coupled continuous-time random walks, which, in turn, is a variation of the well-known Lévy walk [41-46]. In the time interval of observation $(0, t)$, we have $N(t)$ random renewal events, and the pairs of waiting times and velocities are labeled $\left(v_{i}, \tilde{\tau}_{i}\right)$, where $i=1, \ldots, N(t) \quad\left(v_{1}\right.$ is the initial condition). We rewrite the action $\mathcal{S}(t)=\sum_{i=1}^{N(t)} s_{i}+s_{B}(t)$ with $s_{i}=\left(v_{i}\right)^{2} \widetilde{\tau}_{i}$, which is reminiscent of a biased random walk process. The increments $s_{i}>0$ are constrained, since the measurement time is $t=\sum_{i=1}^{N(t)} \tilde{\tau}_{i}+t_{B}(t)$. Here, $t_{B}$ is the backward recurrence time $[47,48]$, the time elapsing between the last update in the process and the measurement time $t$. Similarly, $s_{B}(t)=\left(v_{N(t)+1}\right)^{2} t_{B}(t)$ is the contribution to the action, from the last time interval in the sequence.

To advance the theory, we need the joint PDF of action increments $s$ and waiting times $\tilde{\tau}$ denoted $\phi(s, \tilde{\tau})$, obtained from $\int_{0}^{v_{\max }} d v f(v) \delta\left(s-v^{2} \tilde{\tau}\right) \exp [-\tilde{\tau} / \tau(v)] / \tau(v)$, giving

$$
\phi(s, \tilde{\tau})=\frac{1}{2 v_{\max } \sqrt{s \tilde{\tau}}} R\left(\sqrt{\frac{s}{\tilde{\tau}}}\right) \exp \left[-\tilde{\tau} R\left(\sqrt{\frac{s}{\tilde{\tau}}}\right)\right]
$$

when $0 \leq s \leq v_{\max }^{2} \tilde{\tau}$ and zero otherwise. Here, the waiting times $\tilde{\tau}$ and action increments $s$ are correlated. Integrating over $s$ yields the PDF of the waiting times $\psi(\tilde{\tau})$ whose fat tail reads $\psi(\tilde{\tau}) \sim \operatorname{const} \tilde{\tau}^{-1-\gamma}$ with const $=c^{\gamma} \gamma \Gamma(1+\gamma) / v_{\max }$. The divergence of the mean waiting time for $0<\gamma<1$ signals special ergodic properties [12].

Let $P(\mathcal{S}, t)$ be the PDF of the action at time $t$. The first step in the analysis is to relate this PDF to Eq. (9). Employing the renewal property of the process $[47,49,50]$, we establish this relation using Laplace transforms. Let $\hat{P}(u, p)=\int_{0}^{\infty} \int_{0}^{\infty} d S d t \exp (-u \mathcal{S}-p t) P(\mathcal{S}, t)$ be the double Laplace transform $\mathcal{S} \rightarrow u$ and $t \rightarrow p$ of the $\operatorname{PDF} P(\mathcal{S}, t)$. Then we derive the Montroll-Weiss-like [51] equation [37]

$$
\hat{P}(u, p)=\frac{\hat{\Phi}(u, p)}{1-\hat{\phi}(u, p)} .
$$

Here, $\hat{\phi}(u, p)$ is the double Laplace transform of $\phi(s, \tilde{\tau})$, and $\hat{\Phi}(u, p)$ corresponds to

$$
\Phi\left(s, t_{B}\right)=\int_{0}^{v_{\max }} \frac{d v}{v_{\max }} \exp \left[-R(v) t_{B}\right] \delta\left(s-t_{B} v^{2}\right) .
$$

This term stems from the contribution to the action $s=$ $v^{2} t_{B}$ from the last increment in the sequence, namely, from the backward recurrence time $t_{B}$, while $\exp \left[-R(v) t_{B}\right]$ is the probability of not jolting in that time interval.

Using Eq. (10) to investigate the ergodic properties of the process, we define the dimensionless random variable $\Upsilon=\bar{E}_{k} /\left\langle\bar{E}_{k}\right\rangle$. We first focus on the case when the observable is integrable, namely, $1 / 3<\gamma<1$. In standard ergodic theories, found here if $1<\gamma$, the distribution of $\Upsilon$ will approach a delta function centered on unity. 
By definition, $\Upsilon=\mathcal{S}(t) /\left[t\left\langle\bar{E}_{k}(t)\right\rangle\right]$, and here an analysis of Eq. (10) is useful, since it yields the distribution of $\mathcal{S}$ and then the distribution of the sought-after $\Upsilon$. A detailed analysis shows that the PDF of $\Upsilon$ denoted by $\operatorname{ML}(\Upsilon)$ is given by the universal Mittag-Leffler (ML) law

$$
\operatorname{ML}(\Upsilon)=\frac{[\Gamma(1+\gamma)]^{1 / \gamma}}{\gamma \Upsilon^{1+1 / \gamma}} l_{\gamma, 1}\left(\frac{\left[\Gamma\left(1+\frac{1}{\gamma}\right)\right]^{1 / \gamma}}{\Upsilon^{1 / \gamma}}\right)
$$

Here, $l_{\gamma, 1}($.$) is the one-sided Lévy PDF. This law, which$ replaces Birkhoff's ergodic theory, is a concrete manifestation of the Darling-Kac theorem [14,15]. Our physical approach was to show how this law is related to the lasercooling parameters, i.e., to $\gamma$. This law is valid for any observable of interest, provided that it is integrable; for example, we verified this numerically for the energy observable in Fig. 1 but also for the indicator function. In the limit $\gamma \rightarrow 1, \operatorname{ML}(\Upsilon)$ reduces to a delta function, as expected.

When the energy is a nonintegrable observable, namely, $0<\gamma<1 / 3$, the calculation of the mean energy cannot be made with the infinite density, since the result will diverge. Instead, the ensemble-averaged kinetic energy is found using the scaling solution [Eq. (3)], implying that the energy is now sensitive to the inner part of the velocity packet [37]:

$$
\left\langle E_{k}(t)\right\rangle \sim \frac{\sin (\pi \gamma)}{\sin (3 \pi \gamma)} \frac{1}{\Gamma(1-2 \gamma)} c^{2 \gamma} t^{-2 \gamma} .
$$

This behavior is universal in the sense that this result does not depend on $v_{\max }$, unlike Eq. (8). A simple time integration gives the relation between time and ensemble average $\left\langle\overline{E_{k}(t)}\right\rangle \sim\left\langle E_{k}(t)\right\rangle /(1-2 \gamma)$, which clearly differs from the generic behavior found for integrable observables [Eq. (7)].

To characterize the fluctuations of the time averages, one defines the ergodicity-breaking parameter [52]

$$
\mathrm{EB}=\frac{\left\langle{\overline{E_{k}}}^{2}(t)\right\rangle-\left\langle\overline{E_{k}}(t)\right\rangle^{2}}{\left\langle\overline{E_{k}}(t)\right\rangle^{2}}=\frac{\left\langle\mathcal{S}^{2}(t)\right\rangle-\langle\mathcal{S}(t)\rangle^{2}}{\langle\mathcal{S}(t)\rangle^{2}}
$$

which is zero in the long-time limit, if the process is ergodic, namely, for $\gamma>1$. In the phase where energy is integrable, $\mathrm{EB}=\left[2 \Gamma^{2}(1+\gamma)-\Gamma(1+2 \gamma)\right] / \Gamma(1+2 \gamma)$, for $1 / 3<\gamma<1$, which is universal in the sense of going beyond the observable of interest. For example, this describes also the fluctuations of the number of renewals $N(t)$.

Returning to the case $\gamma<1 / 3$, we find the second moment of the action, obtained in Laplace space after differentiation of Eq. (10):
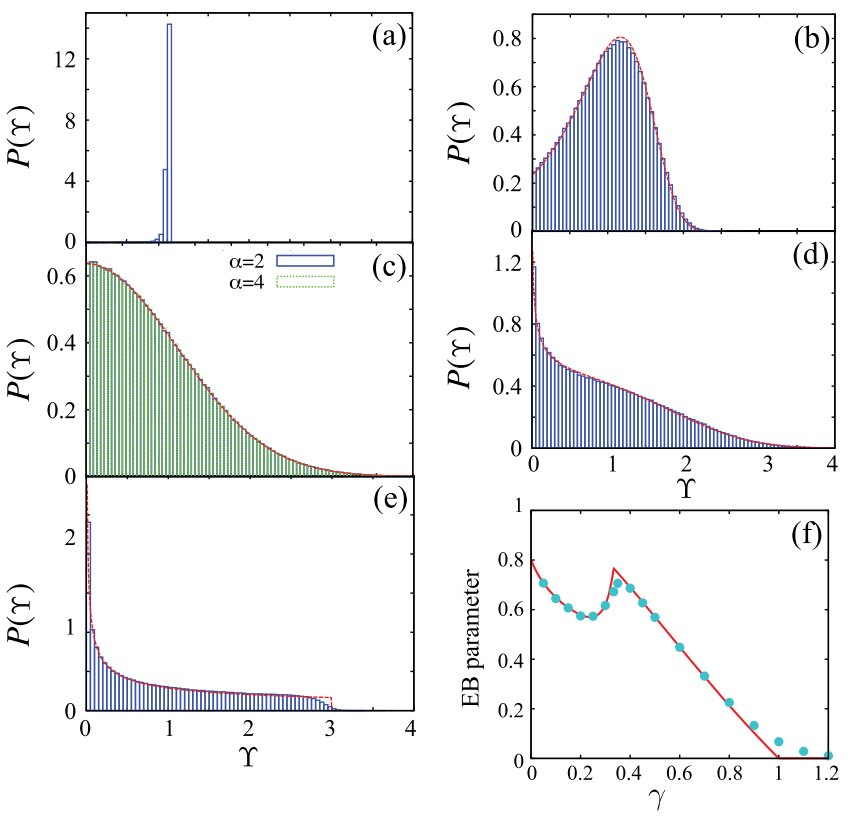

FIG. 1. In the process of subrecoil-laser cooling, the PDF $P(\Upsilon)$ of the time-averaged energy $\Upsilon=\overline{E_{k}(t)} /\left\langle\overline{E_{k}(t)}\right\rangle$ exhibits a wide range of physical behaviors. When $\gamma=1 / \alpha>1$, ergodicity holds and the PDF approaches a delta function in the long-measurement-time limit; see finite time simulations in (a) with $\alpha=0.8$ (details in Ref. [37]). When $\alpha=1.25$, the energy is integrable with respect to the infinite measure, and $P(\Upsilon)$ is nontrivial though it has a peak close to unity, since at this stage we are not too far from the ergodic phase (b). For $\alpha=2$ and $\alpha=4$, we find that the distributions are nondistinguishable and described by a half-Gaussian, presented in (c). Here, the energy observable is integrable in the first case, while it is not in the second; further note that the Mittag-Leffler distribution is half-Gaussian when $\alpha=2$, since $\gamma=1 / 2$. When $\alpha=6$, the energy observable is nonintegrable and $P(\Upsilon)$ diverges for $\Upsilon \rightarrow 0$, indicating very large deviations from usual ergodic behavior (d). In the limit $\alpha \rightarrow \infty$, we get a discontinuous behavior, a sharp cutoff at $\Upsilon=3$ [see (e) and simulation with $\alpha=50$ ]. Finally, the EB parameter (f) exhibits a cusp at $\gamma=1 / \alpha=1 / 3$. This value marks a transition for the ergodic behavior of the system, from an energy that is integrable with respect to the infinite measure to nonintegrable. In the figure, simulations match theoretical predictions without fitting.

$$
\left\langle\hat{\mathcal{S}}^{2}(p)\right\rangle=\left.\frac{\partial^{2}}{\partial u^{2}} \frac{\hat{\Phi}(u, p)}{1-\hat{\phi}(u, p)}\right|_{u=0} .
$$

We find asymptotically, for large times and for $0<\gamma<1 / 3$,

$\mathrm{EB}=\frac{2 \Gamma^{2}(2-2 \gamma)}{\Gamma(3-4 \gamma)}\left[\frac{\sin ^{2}(3 \pi \gamma)(1-5 \gamma)}{\sin (\pi \gamma) \sin (5 \pi \gamma)}+3 \gamma\right]-1$

This expression clearly differs from the EB parameter found in the Darling-Kac phase $1 / 3<\gamma<1$. When $\gamma \rightarrow 0$, we find $\mathrm{EB}=4 / 5$. In this limit, the particle maintains a constant 
velocity for (nearly) all the observation time, so $\bar{E}_{k}=v^{2}$ and, using the uniform $f(v), \lim _{\gamma \rightarrow 0} \mathrm{~EB}=$ $\left(\left\langle v^{4}\right\rangle-\left\langle v^{2}\right\rangle^{2}\right) /\left\langle v^{2}\right\rangle^{2}=4 / 5$. The EB parameter versus $0<\gamma<1$ is plotted in Fig. 1(f), and it exhibits a clear transition when $\gamma=1 / 3$. Thus, switching from the case when energy is integrable to nonintegrable manifests itself in nontrivial fluctuations of the time averages.

We have investigated semianalytically the distribution of the time averages also in the nonintegrable phase $\gamma<1 / 3$, namely, $\alpha>3$. Here, the Mittag-Leffler law is not valid anymore. In Fig. 1, we present some of the main results of this mathematically challenging domain. For example, we find $\lim _{\gamma \rightarrow 0} P(\Upsilon)=\Upsilon^{-1 / 2} /(2 \sqrt{3})$ for $\Upsilon<3$; otherwise, $P(\Upsilon)=0$. This result can be explained, as for the EB parameter, by noting that the atom maintains a constant velocity for practically all the duration of the measurement; i.e., in this limit $\Upsilon=v^{2} /\left\langle v^{2}\right\rangle$, and the PDF of $v$ is the uniform $f(v)$. For the experimentally relevant case $\alpha=4$ $(\gamma=1 / 4)$, we find that the distribution of $\Upsilon>0$ is halfGaussian; see Fig. 1(c). Interestingly, this case also marks a transition: The PDF of $\Upsilon$ diverges at the origin for any $\alpha>4$ and vanishes there for $3<\alpha<4$. This means that for $\alpha>4$ the most likely time average is found for cases where it is much smaller than the ensemble average. Finally, for $\alpha=6$ [33], we find the solution in terms of a Fox-H function [39]:

$$
P(\Upsilon) \simeq \frac{C}{\Gamma\left(\frac{3}{4}\right)} H_{1,1}^{1,0}\left(\begin{array}{l|l}
C \Upsilon & \begin{array}{c}
\left(\frac{1}{3}, \frac{2}{3}\right) \\
\left(-\frac{1}{4}, 1\right)
\end{array}
\end{array}\right)
$$

with $C=3 / 4 \Gamma(5 / 3)$, which perfectly matches the simulation presented in Fig. 1(d).

What are the consequences for cooling? Remarkably, using Eqs. (7), (8), and (13), we conclude that the most efficient cooling, in the sense of the fastest relaxation of the mean energy, is found for $\alpha=3$. Thus, the transition in the ergodic properties of the system investigated here is physically connected to optimal cooling of energy. In contrast, for the FWHM of the velocity packet [34], we do not have such an optimum. Thus, the classification of an observable as either integrable (energy $\alpha<3$ ) or nonintegrable (energy $\alpha>3$, FWHM) with respect to the infinite invariant measure is crucial, both mathematically and physically. Moreover, we discovered for $\alpha>4$ an accumulation effect, namely, the divergence of the PDF of the time averages, found at low energies, e.g., Fig. 1(d), where $\alpha=6$ and $\Upsilon \rightarrow 0$. This means that a significant population of atoms remains at small velocities for the whole duration of the experiment. In turn, this is useful when one wishes to reduce scattering or spatial spreading, namely, holding atoms close to the dark zero momentum state for long durations. Thus, while for the optimization of relaxation time of the FWHM, which decays as $t^{-1 / \alpha}$, one should consider small values of $\alpha$ to obtain fast relaxation (say, $\alpha=2$ ), to maintain some of the population with small kinetic energy for long durations, large values of $\alpha$ (say, $\alpha=6$ ) are beneficial, as the trapping times become statistically longer. Surprisingly, $\alpha=4$ marks a quantitative transition of the low-energy statistics, which we discovered from the analysis of the time averages.

The support of Israel Science Foundation's Grant No. $1898 / 17$ is acknowledged (E. B.). This work was supported by the JSPS KAKENHI Grant No. 240 18K03468 (T. A.). We thank Tony Albers, Nir Davidson, and Lev Khaykovich for helpful suggestions.

[1] A. Aspect, E. Arimondo, R. Kaiser, N. Vansteenkiste, and C. Cohen-Tannoudji, Laser Cooling below the One-Photon Recoil Energy by Velocity-Selective Coherent Population Trapping, Phys. Rev. Lett. 61, 826 (1988).

[2] M. Kasevich and S. Chu, Laser Cooling below a Photon Recoil with Three-Level Atoms, Phys. Rev. Lett. 69, 1741 (1992).

[3] N. Davidson, H. J. Lee, M. Kasevich, and S. Chu, Raman Cooling of Atoms in Two and Three Dimensions, Phys. Rev. Lett. 72, 3158 (1994).

[4] F. Bardou, J. P. Bouchaud, O. Emile, A. Aspect, and C. Cohen-Tannoudji, Sub-Recoil Laser Cooling and Lévy Flights, Phys. Rev. Lett. 72, 203 (1994).

[5] S. Marksteiner, K. Ellinger, and P. Zoller, Anomalous diffusion and Lévy walks in optical lattices, Phys. Rev. A 53, 3409 (1996).

[6] F. Bardou, J. P. Bouchaud, A. Aspect, and C. CohenTannoudji, Lévy Statistics and Laser Cooling: How Rare Events Bring Atoms to Rest (Cambridge University Press, Cambridge, England, 2002).

[7] Y. Sagi, M. Brook, I. Almog, and N. Davidson, Observation of Anomalous Diffusion and Fractional Self-Similarity in One Dimension, Phys. Rev. Lett. 108, 093002 (2012).

[8] D. A. Kessler and E. Barkai, Theory of Fractional-Lévy Kinetics for Cold Atoms Diffusing in Optical Lattices, Phys. Rev. Lett. 108, 230602 (2012).

[9] A. Dechant and E. Lutz, Anomalous Spatial Diffusion and Multifractality in Optical Lattices, Phys. Rev. Lett. 108, 230601 (2012).

[10] E. Barkai, E. Aghion, and D. Kessler, From the Area under the Bessel Excursion to Anomalous Diffusion of Cold Atoms, Phys. Rev. X 4, 021036 (2014).

[11] E. Lutz and F. Renzoni, Beyond Boltzmann-Gibbs statistical mechanics in optical lattices, Nat. Phys. 9, 615 (2013).

[12] J. Bouchaud, Weak ergodicity breaking and aging in disordered systems, J. Phys. I, EDP Sci. 2, 1705 (1992).

[13] B. Saubaméa, M. Leduc, and C. Cohen-Tannoudji, Experimental Investigation of Non-Ergodic Effects in Subrecoil Laser Cooling, Phys. Rev. Lett. 83, 3796 (1999).

[14] D. A. Darling and M. Kac, On occupation times for Markoff process, Trans. Am. Math. Soc. 84, 444 (1957).

[15] J. Aaronson, An Introduction to Infinite Ergodic Theory (American Mathematical Society, Providence, 1997). 
[16] R. Zweimuller, Surrey Notes on Infinite Ergodic Theory, Lecture Notes (Surrey University, Guildford, England, 2009).

[17] N. Korabel and E. Barkai, Pesin-Type Identity for Intermittent Dynamics with a Zero Lyapunov Exponent, Phys. Rev. Lett. 102, 050601 (2009).

[18] D. Kessler and E. Barkai, Infinite Covariant Density for Diffusion in Logarithmic Potentials and Optical Lattices, Phys. Rev. Lett. 105, 120602 (2010).

[19] T. Akimoto and T. Miyaguchi, Role of infinite invariant measure in deterministic subdiffusion, Phys. Rev. E 82, 030102(R) (2010).

[20] T. Akimoto, Distributional Response to Biases in Deterministic Super-Diffusion, Phys. Rev. Lett. 108, 164101 (2012).

[21] P. C. Holz, A. Dechant, and E. Lutz, Infinite density for cold atoms in shallow optical lattices, Europhys. Lett. 109, 23001 (2015).

[22] T. Akimoto, S. Shinkai, and Y. Aizawa, Distributional behavior of time averages of non- $L^{1}$ observables in onedimensional intermittent maps with infinite invariant measures, J. Stat. Phys. 158, 476 (2015).

[23] P. Meyer and H. Kantz, Infinite invariant densities due to intermittency in a nonlinear oscillator, Phys. Rev. E 96, 022217 (2017).

[24] A. Vezzani, E. Barkai, and R. Burioni, Single-big-jump principle in physical modeling, Phys. Rev. E 100, 012108 (2019).

[25] E. Aghion, D. A. Kessler, and E. Barkai, From NonNormalizable Boltzmann-Gibbs Statistics to InfiniteErgodic Theory, Phys. Rev. Lett. 122, 010601 (2019).

[26] E. Aghion, D. A. Kessler, and E. Barkai, Infinite ergodic theory meets Boltzmann statistics, Chaos, Solitons \& Fractals 138, 109890 (2020).

[27] Y. Sato and R. Klages, Anomalous Diffusion in Random Dynamical Systems, Phys. Rev. Lett. 122, 174101 (2019).

[28] L. Defaveri, C. Anteneodo, D. Kessler, and E. Barkai, Regularized Boltzmann Gibbs statistics for a Brownian particle in a nonconfining field, Phys. Rev. Research 2, 043088 (2020).

[29] T. Akimoto, E. Barkai, and G. Radons, Infinite invariant density in a semi-Markov process with continuous state variables, Phys. Rev. E 101, 052112 (2020).

[30] M. Radice, M. Onofri, R. Artuso, and G. Pozzoli, Statistics of occupation times and connection to local properties of non-homogeneous random walks, Phys. Rev. E 101, 042103 (2020).

[31] C. Streißnig and H. Kantz, Work fluctuation theorem for a Brownian particle in a nonconfining potential, Phys. Rev. Research 3, 013115 (2021).

[32] O. Farago, The thermodynamics of a Brownian particle in a non-confining potential, Phys. Rev. E 104, 014105 (2021).

[33] E. Bertin and F. Bardou, From laser cooling to aging: A unified Lévy flight description, Am. J. Phys. 76, 630 (2008).

[34] J. Reichel, F. Bardou, M. Ben Dahan, E. Peik, S. Rand, C. Salomon, and C. Cohen-Tannoudji, Raman Cooling of
Cesium below 3 nK: New Approach Inspired by Lévy Flight Statistics, Phys. Rev. Lett. 75, 4575 (1995).

[35] J. Reichel, Refroidissement Raman et vols de Lévy: Atomes de césium au nanoKelvin (Physique Atomique, Université Pierre et Marie Curie-Paris VI, Paris, 1996), in French.

[36] C. Cohen-Tannoudji and D. Guéry-Odelin, Sub-Doppler cooling: Sub-recoil cooling, in Advances in Atomic Physics an Overview (World Scientific, Singapore, 2011), Chap. 13, pp. 291-315, https://doi.org/10.1142/9789812774989_0013.

[37] See Supplemental Material at http://link.aps.org/supplemental/ 10.1103/PhysRevLett.127.140605 for a brief derivation of the infinite density, the scaling solution, and the Montroll-Weiss equation, which includes Refs. [6,33,38,39].

[38] A. D. Polyanin and A. V. Manzhirov, Handbook of Integral Equations, 2nd ed. (Chapman \& Hall/CRC, Boca Raton, 2008).

[39] A. P. Prudnikov, Yu. A. Brychkov, and O. I. Marichev, More Special Functions, Integrals and Series Vol. 3 (Gordon and Breach, New York, 1989).

[40] Start of measurement could be some $t_{a}>0$, and, as long as $t \gg t_{a}$, our results below are valid.

[41] J. Klafter, A. Blumen, and M. F. Shlesinger, Stochastic pathway to anomalous diffusion, Phys. Rev. A 35, 3081 (1987).

[42] R. Metzler and J. Klafter, Random walk's guide to anomalous diffusion: A fractional dynamics, Phys. Rep. 339, 1 (2000).

[43] T. Akimoto and T. Miyaguchi, Phase diagram in storedenergy-driven Lévy flight, J. Stat. Phys. 157, 515 (2014).

[44] R. Kutner and J. Masoliver, The continuous time random walk, still trendy: Fifty-year history, state of art and outlook, Eur. Phys. J. B 90, 50 (2017).

[45] V. Zaburdaev, S. Denisov, and J. Klafter, Lévy walks, Rev. Mod. Phys. 87, 483 (2015).

[46] T. Albers and G. Radons, Exact Results for the Nonergodicty of d-Dimensional Generalized Lévy Walks, Phys. Rev. Lett. 120, 104501 (2018).

[47] C. Godrèche and J. M. Luck, Statistics of the occupation time of renewal processes, J. Stat. Phys. 104, 489 (2001).

[48] C. Godréche, S. Majumdar, and G. Schehr, Statistics of the longest interval in renewal processes, J. Stat. Mech. 03 (2015) P03014.

[49] J. E. Santos, T. Franosch, A. Parmeggiani, and E. Frey, Renewal processes and fluctuation analysis of molecular motor stepping, Phys. Biol. 2, 207 (2005).

[50] D. Daley and D. Vere-Jones, An Introduction to the Theory of Point Processes, Vol. I Elementary Theory and Methods (Springer, New York, 2003).

[51] E. W. Montroll and G. H. Weiss, Random walks on lattices. II, J. Math. Phys. (N.Y.) 6, 167 (1965).

[52] Y. He, S. Burov, R. Metzler, and E. Barkai, Random TimeScale Invariant Diffusion and Transport Coefficients, Phys. Rev. Lett. 101, 058101 (2008). 